\title{
Diagnosis and management of central hypersomnias
}

\author{
Karel Sonka and Marek Susta
}

\begin{abstract}
Central hypersomnias are diseases manifested in excessive daytime sleepiness (EDS) not caused by disturbed nocturnal sleep or misaligned circadian rhythms. Central hypersomnias includes narcolepsy with and without cataplexy, recurrent hypersomnia, idiopathic hypersomnia, with and without long sleep time, behaviorally induced insufficient sleep syndrome, hypersomnia and narcolepsy due to medical conditions, and finally hypersomnia induced by substance intake. The Epworth Sleepiness Scale is a subjective tool mostly used for EDS assessment, while the Multiple Sleep Latency Test serves as an objective diagnostic method for narcolepsy and idiopathic hypersomnias. As for symptomatic therapy of EDS, the central nervous system stimulants modafinil and methylphenidate seem to work well in most cases and in narcolepsy and Parkinson's disease; sodium oxybate also has notable therapeutic value.
\end{abstract}

Keywords: Excessive daytime sleepiness, hypersomnia, methylphenidate, modafinil, narcolepsy, sodium oxybate

\section{Introduction}

The International classification of sleep disorders second edition [American Academy of Sleep Medicine, 2005a] describes hypersomnias of central origin as the group of disorders in which the primary complaint is excessive daytime sleepiness (EDS), which is not caused by disturbed nocturnal sleep or misaligned circadian rhythms. This group of diseases includes narcolepsy with cataplexy, narcolepsy without cataplexy, recurrent hypersomnia, idiopathic hypersomnia with long sleep time, idiopathic hypersomnia without long sleep time, behaviorally induced insufficient sleep syndrome, hypersomnia and narcolepsy due to medical conditions, and hypersomnia due to drug or substance intake. This article deals with narcolepsy, idiopathic and recurrent hypersomnia. There are also a number of notes regarding the group of hypersomnias due to medical conditions, namely excessive sleepiness in Parkinson's disease (PD) and other diseases with Parkinsonism, multiple sclerosis (MS), traumatic brain injury (TBI), and in stroke.

\section{Excessive Daytime Sleepiness}

EDS is defined as the reduced ability to stay awake and alert during normal daytime hours, resulting in lapses of sleepiness or sleep. EDS seriously decreases quality of life for affected patients [Ozaki et al. 2012]. Depending on their personality type, occupation, social status, and other circumstances, patients' symptoms can vary in intensity and inconvenience.

For the diagnosis of central hypersomnias (with the exception of recurrent hypersomnia and hypersomnia due to drug and substance intake), EDS symptoms must be present for at least 3 months. All information on sleepiness as reported by the patients or their families requires correction by the physician's clinical experience. Some typical variants of EDS prompt the final diagnosis. Irresistible short refreshing naps are typical for narcolepsy. Long daytime sleep ended by forced awakening after which the patient feels less alert and refreshed even before taking the nap is typical for idiopathic hypersomnia with long sleep time. Sleep attacks without any warning signs are typical for narcolepsy [Dauvilliers et al. 2007]. The same symptoms are experienced under the influence of dopaminergic drugs used in PD [Frucht et al. 1999]. Sleepiness can result in an automatic continuation of activity in a semiconscious state with no memory of the incident. This phenomenon is frequent in narcolepsy. Precise
Ther Adv Neurol Disord

(2012) 5(5) 297-305

DOI: $10.1177 /$

1756285612454692

(c) The Author(s), 2012

Reprints and permissions: http://www.sagepub.co.uk/ journalsPermissions.nav
Correspondence to: Karel Sonka, MD, DSc Department of Neurology and Center of Clinical Neuroscience, First Faculty of Medicine, Charles University in Prague and General University Hospital in Prague, Katerinska 30 Prague, Czech Republic ksonkaAlf1.cuni.cz

Marek Susta, PhD Department of Psychiatry, First Faculty of Medicine, Charles University in Prague and General University Hospital in Prague, Prague, Czech Republic 
description of daytime sleepiness is very important for diagnostic process despite the fact that the only central hypersomnia where diagnosis is based solely on daytime sleepiness pattern is recurrent hypersomnia. Diagnosis of all of the other primary hypersomnias also requires the presence of other symptoms or special examination. The Epworth Sleepiness Scale is mostly used for subjective quantitative rating of sleepiness [Johns, 1991]. The Epworth Sleepiness Scale value of 10 and higher is considered abnormal. Other symptoms noted in particular diseases help greatly in the diagnostic process. The basic diagnostic protocol in EDS consists of nocturnal polysomnography and the Multiple Sleep Latency Test (MSLT). Nocturnal polysomnography should precede MSLT in standard settings [Carskadon et al. 1986] to assure the validity of MSLT (objectivization of the previous night's sleep length and quality); night sleep information also helps with diagnosis. MSLT is indicated in suspected narcolepsy and idiopathic hypersomnia [American Academy of Sleep Medicine, 2005b] and in EDS differential diagnosis. In differential diagnosis, central hypersomnias caused by psychiatric disorders or psychoactive drugs (classified and described elsewhere) must also be taken into account [Ivanenko, 2008; Winkelman and Plante, 2010].

\section{General aspects of EDS treatment and the most important of the available drugs}

While the body of practical experience of treatment for narcolepsy keeps growing, robust drug studies of EDS treatment except for narcolepsy are still missing. Any therapy must be preceded by clinical examination and identification of its cause. Symptomatic treatment should be resorted to situations when all other options for causal treatment, regimen adjustments such as sleep hygiene and planned naps namely in narcolepsy patients have been exhausted. The following groups of drugs are used for EDS control:

- conventional central nervous system (CNS) stimulants (amphetamine and its derivatives including methylphenidate, dextroamphetamine and pemoline);

- nonamphetamine CNS stimulants (modafinil and armodafinil);

- sodium oxybate;

- caffeine;

- antidepressants with stimulant properties (e.g. atomoxetine);
- monoamine oxidase (MAO) inhibitors with alerting effect (e.g. selegiline);

- the dopamine/norepinephrine uptake inhibitor mazindol;

- new drugs in clinical trials (e.g. pitolisant and hypocretin-1).

\section{CNS stimulants}

Amphetamine and amphetamine-like compounds enhance catecholaminergic transmission (dopaminergic, in particular) and, in higher doses, other mechanisms start to play a role, including interaction with monoamine transporters. Manifestations of overdose (anxiety, headache, motor hyperactivity, failure to concentrate, tremor, aggressiveness, anorexia, blood pressure increase, etc.) do not occur sporadically. Psychotic symptoms may also appear, although rarely. In many countries, amphetamines are no longer marketed because of their potential for abuse. Pemoline has often been used in the past; however, cases of lethal hepatotoxicity have resulted in its withdrawal from the market in many countries.

Methylphenidate is a piperazine derivative of amphetamine working also by means of catecholaminergic transmission activation. Methylphenidate is known for its relatively mild side effects and for fairly short half-life of 2-7 hours, which is why it can also be used on demand. It is regularly used in oral doses of $10-60 \mathrm{mg} /$ day in 1-3 daily doses (maximum single dose of $20 \mathrm{mg}$ ). Methylphenidate is also available in the sustained-release form. The safety profile of methylphenidate is better than that of amphetamine but there are no reliable safety studies of methylphenidate.

\section{Nonamphetamine CNS stimulants}

Modafinil is the most common drug used in sleepiness therapy. Exactly how it works remains unclear; it is supposed that it acts by blocking norepinephrine and dopamine re-uptake transporters. Its plasma concentration will reach peak values within 2-4 hours after intake. Modafinil has a linear pharmacokinetic profile with a halflife of elimination of 9 up to 14 hours. It is completely metabolized in the liver down to inert metabolites which are excreted mainly in urine. Modafinil is safe and well tolerated, its most frequent undesirable effects (headache, nausea, loss of appetite and nervousness) are infrequent and seldom lead to therapy refusal [Roth et al. 2007]. In terms of side effects, tolerance and addiction 
risk, long-term experience of its administration to narcoleptics is encouraging. However, there is also clinical experience that shows, in some patients, need for dosage increase after a longterm use. Although modafinil is a P450 enzyme inductor, the effectiveness of steroidal contraceptives may be reduced when used in combination with modafinil thus alternative or concomitant methods of contraception are recommended. Because modafinil is a reversible inhibitor of the drug-metabolizing enzyme CYP2C19, coadministration of modafinil with drugs such as diazepam, phenytoin and propranolol may increase the circulating levels of those compounds. In addition, individuals deficient in the CYP2D6 enzyme (i.e. $7-10 \%$ of the White population; similar or lower in other populations), the levels of CYP2D6 substrates such as tricyclic antidepressants and selective serotonin reuptake inhibitors, metabolized by CYP2C19, may be increased by co-administration of modafinil [Food and Drug Administration, 2012]. In the European Union the use of modafinil has been restricted recently to only narcoleptic adults because of reports of serious skin and allergic reactions. The age limitation has been criticized by an expert group based on their own experience [Lecendreux et al. 2012]. In opposition to the European Union recommendation, modafinil was found to be as efficient and safe in idiopathic hypersomnia as in narcolepsy [Lavault et al. 2011]. Armodafinil [Lankford, 2008] is a recently developed R-enantiomer of modafinil with a prolonged action and similar efficacy and safety profile. Armodafinil therapy requires lower dosage than modafinil and needs to be taken only once a day.

\section{Sodium oxybate}

The pharmacological form of gammahydroxybutyrate, sodium oxybate, exerts a favorable effect on alertness in narcoleptics. Administered in pharmacological doses, sodium oxybate appears to be an agonist of a newly discovered gammahydroxybutyrate (excitatory) receptor and a weak GABA B (inhibitory) receptor agonist, consolidating nocturnal sleep by reducing fragmentation and improving its quality. The recommended dosage of sodium oxybate is $4.5-9 \mathrm{~g}$ daily taken in two doses: one immediately before going to bed, the other 2.5-4 hours thereafter. While there are no known serious pharmacological interactions of sodium oxybate, alcohol and other centrally acting inhibitors are strictly forbidden and sodium oxybate is not recommended in sleep apnea. Sodium oxybate is known for its central inhibitory effects and for its potential to induce dependence and abuse. Gammahydroxybutyrate is misused in athletes for its metabolic effect (growth hormone releasing effect) and it has been used as a 'date rape' drug because of its rapid sedating effect. However, sodium oxybate has a very low risk in narcolepsy patients. As many studies suggest, the risk of undesirable effects in the treatment of narcolepsy with cataplexy is at most moderate. The drug can be combined with modafinil [Boscolo-Berto et al. 2011]. Sodium oxybate is registered for the treatment of narcolepsy with cataplexy in Europe [European Medicines Agency, 2005] and in the US for treatment of cataplexy and EDS caused by narcolepsy.

\section{Caffeine}

Caffeine is a xanthine derivative and nonspecific antagonist of adenosine receptors. Adenosine is an interesting neurotransmitter, the levels of which increase with prolonged wakefulness. Caffeine's stimulatory effect is rather mild. It is mostly taken in the form of a drink but it exists also in a tablet form sold in pharmacies. Twice daily doses of $100 \mathrm{mg}$ seem to have a rather favorable effect/side effect ratio.

\section{Selegiline}

Selegiline is a selective irreversible MAO B inhibitor, which is metabolized into various compounds, including amphetamine and methamphetamine. Dietary restrictions, incompatibility with triptans and selective serotonin reuptake inhibitors and tricyclic antidepressants limit its routine use.

\section{Mazindol}

Mazindol is rarely used due to its side effects (e.g. nervousness, tachycardia, dry mouth, anorexia). It is no longer marketed in many countries.

\section{New drugs}

Pitolisant is the inverse agonist of the $\mathrm{H} 3$ receptor and its wake-promotion activity was evidenced in excessive diurnal sleepiness of patients with narcolepsy and other EDS diseases decreasing mean of the Epworth Sleepiness Scale in average by five units [Schwartz, 2011]. 
Hypocretin-1 administered by intranasal route has functional effects on sleep in narcolepsy with cataplexy [Baier et al. 2011] and is one of the promising future drugs.

While the list of medications is not absolute and, due to ongoing research is constantly updated, the most commonly used drugs have been covered here. A more detailed description of use and dosage is listed in the following section focusing on particular nosologic entities.

\section{Narcolepsy}

Narcolepsy is a disabling sleep disorder characterized by EDS and abnormal rapid-eye movement (REM) sleep manifestations, including cataplexy, sleep paralysis, hypnagogic hallucinations and sleep onset REM periods. Narcolepsy with cataplexy has a prevalence of $0.02-0.067 \%$. The loss of hypothalamic hypocretin-producing neurons causes narcolepsy with cataplexy. An autoimmune basis for narcolepsy with cataplexy has long been suspected and recent results have greatly strengthened this hypothesis. Narcolepsy with cataplexy and with hypocretin deficiency is now known to be associated with a human leukocyte antigen (HLA) and T-cell receptor (TCR) polymorphisms, suggesting that an autoimmune process targets a single peptide unique to hypocretin cells via specific HLA-peptide-TCR interactions. Recent data have shown a robust seasonality of disease onset in children and associations with Streptococcus pyogenes, and influenza A H1N1 infection and $\mathrm{H} 1 \mathrm{~N} 1$ vaccination, pointing towards processes such as molecular mimicry or bystander activation as crucial for disease development [Kornum et al. 2011]. Etiopathogenesis of narcolepsy without cataplexy is not clear.

EDS is the most inconveniencing symptom of both forms of narcolepsy, the objective measure of which is mean sleep latency $<8 \mathrm{~min}$ at MSLT. The second MSLT diagnostic criterion is the presence of two or more sleep-onset REM periods (SOREMPs) [American Academy of Sleep Medicine, 2005a]. The MSLT can fail to document both narcolepsy criteria and if repeated the probability to confirm the diagnosis is high enough [Coelho et al. 2011]. Night sleep is disturbed by PLMS and REM sleep behavior disorder. Narcolepsy with cataplexy has low or undetectable levels of hypocretin in cerebrospinal fluid [Mignot et al. 2002]. Cerebrospinal fluid hypocretin examination is not mandatory for diagnosis of narcolepsy with cataplexy but is found to be very helpful in patients on antidepressants in which it is unlikely that a history of clearcut cataplexy and SOREMPs in MSLT will be found. Cerebrospinal fluid hypocretin in narcolepsy without cataplexy is in the normal range. HLA subtype DQB $1^{\star} 06: 02$ is positive in $95 \%$ of narcolepsy with cataplexy, but only in $40 \%$ of narcolepsy without cataplexy. Because this allele is positive also in $18-35 \%$ of the general population this investigation only has supportive value for diagnosing narcolepsy with cataplexy [Mignot $e t$ al. 1997]. Narcolepsy with cataplexy is associated with higher BMI [Sonka et al. 2010].

A short nap prevents unwanted sleep in narcolepsy, thus scheduled short naps are advised as nonpharmacologic EDS treatment: the optimal nap time schedule and duration is individual. According to four level-one studies, modafinil is effective for the treatment of EDS due to narcolepsy [Billiard et al. 1994; Broughton et al. 1997; US Modafinil in Narcolepsy Multicenter Study Group, 1998, 2000] and modafinil is the first-line drug in the treatment of EDS in narcolepsy. The recommended dose of modafinil ranges from one dose of $200 \mathrm{mg}$ up to $400 \mathrm{mg}$, or better, in a splitdose regimen (early morning and noon) [Billiard et al. 2006; Morgenthaler et al. 2007; Wise et al. 2007]. According to two open-label studies, modafinil is effective and well tolerated in children [Morgenthaler et al. 2007]. Armodafinil, the longer half-life enantiomer of modafinil, given in doses of 250 and $150 \mathrm{mg}$, also produced improved wakefulness in adult narcoleptics [Harsh et al. 2006]. Similarly, sodium oxybate was found to be effective in the treatment of narcolepsy EDS at a dose of $9 \mathrm{~g} / \mathrm{night}$, as shown in three level-one studies. The point is that the anti-EDS effect of sodium oxybate will not appear immediately and a number of weeks of treatment are required. Sodium oxybate is immediately effective in the control of cataplexy and for nocturnal sleep improvement (see the more important publications of level-one studies [Xyrem International Study Group, 2005; Xyrem Multicenter Study Group, 2002, 2003, 2004] and the meta analysis article [BoscoloBerto et al. 2011]). Sodium oxybate and modafinil are both effective for treating EDS in narcolepsy, producing additional effects when used together. Years of clinical practice, as well as two leveltwo studies and one level-three study (all of short duration), justify the use of amphetamine, methamphetamine, dextroamphetamine and especially methylphenidate [Littner et al. 2001; Black and 
Houghton, 2006; Wise et al. 2007]. Despite a lack of information on dependence development in patients with narcolepsy, this risk should be taken into account. Tolerance toward amphetamine and its derivatives is likely to develop in one third of the cases.

The evidence of efficiency of sodium oxybate is well documented in the treatment of cataplexy [Boscolo-Berto et al. 2011]. Antidepressants are also used for the treatment of cataplexy despite poor evidence of their effect and safety. The most commonly used antidepressants are tricyclic (clomipramine, imipramine), selective serotonin reuptake inhibitors (fluoxetine, es/citalopram, fluvoxamine) and selective serotonin and norepinephrine reuptake inhibitors (venlafaxine and atomoxetine), but controlled studies are scarce in this area. The side effect profile of selective serotonin reuptake inhibitors and tricyclic antidepressants includes sexual difficulties and in cases where a high dosage of tricyclics is used also anticholinergic properties. Nevertheless, in many patients a small dosage of $25 \mathrm{mg}$ of clomipramine a day or less is sufficient.

Fragmented nocturnal sleep is improved by sodium oxybate [Black et al. 2010], other therapeutic options (e.g. zolpidem) have no published evidence of effect. Hypnagogic hallucinations, sleep paralysis and vivid dreams are improved by sodium oxybate and by antidepressants. REM sleep behavior disorder is treated by clonazepam, alternative therapy represents melatonin, although the therapeutic effect of melatonin is supported only by studies with small patient groups.

Because a large number of findings suggest that narcolepsy has an autoimmune basis, the intravenous immunoglobulins were administered in some cases of narcolepsy with cataplexy. The results are partly promising [Dauvilliers et al. 2009] but not consistent and a controlled study is needed.

\section{Idiopathic hypersomnia}

Patients suffering from idiopathic hypersomnia with long sleep time almost never feel fully alert despite a normal or long night's sleep. They typically sleep much longer on weekends than on working days. In the morning, they need somebody else to wake them up and the awakening is frequently accompanied by sleep inertia. Most of the patients do not feel refreshed after short naps. They are more frequently evening types. These patients are able to focus only for a limited time. Mental fatigability, dependence on other people to wake them up and a reduced benefit from usually alerting conditions (except being hyperactive or stressed) seem to be more specific of the daily problems of patients with hypersomnia with long sleep time than daytime sleepiness [Vernet et al. 2010]. The symptoms of hypersomnia without long sleep time are limited only to daytime sleepiness. Sleepiness must be documented by mean sleep latency $<8 \mathrm{~min}$ at MSLT in both types of idiopathic hypersomnia and the number of SOREMPs cannot exceed one [American Academy of Sleep Medicine, 2005a].

Treatment of idiopathic hypersomnia has never been tested in level-one and level-two drug studies due to its rare occurrence (10 times less frequent than narcolepsy). In practice, CNS stimulants are used with success, in particular methylphenidate and modafinil at dosages similar to those in narcolepsy [Ali et al. 2009; Lavault et al. 2011; Morgenthaler et al. 2007].

\section{Recurrent hypersomnia}

The diagnostic criteria of recurrent hypersomnia include EDS episodes of 2-28 days duration occurring at least once a year. Between attacks of sleepiness the patient is symptom free. Clinical subtypes are Kleine-Levin syndrome and menstrual related hypersomnia. Kleine-Levin syndrome is characterized by hypersomnia episodes associated with compulsive eating and/or other behavioral abnormalities such as sexual disinhibition and/or odd behavior, cognitive abnormalities such as derealization, confusion, delusions/ hallucinations and psychiatric symptoms such as depression and/or anxiety. Menstrual-related hypersomnia is characterized by recurrent episodes of hypersomnia and/or other symptoms of KleineLevin syndrome, associated with menstruation and/or puerperium [American Academy of Sleep Medicine, 2005a; Billiard et al. 2011].

Reports on therapy of recurrent hypersomnia are rare. In some cases stimulants calmed down symptoms; lithium was the only drug able to prevent attacks of sleepiness in multiple cases [Arnulf et al. 2005; Poppe et al. 2003].

\section{Parkinson's disease and Parkinsonism}

Patients with PD and other diseases with Parkinsonism (dementia with Lewy body disease, 
multisystem atrophy) suffer frequently from daytime sleepiness (for PD at a rate of $15-50 \%$ ).

The PD pathologies affect many neurotransmitter systems involved in wakefulness and thus appear to be the greatest contributors to EDS in PD. Considering that PD and Parkinsonism may primarily be associated with sleep apnea, restless legs syndrome, periodic limb movements in sleep and with insufficient or interrupted sleep of other identifiable reasons, night sleep must be examined in cases of any disturbances of daytime wakefulness. Dopaminergic drugs exacerbate sleepiness in a dose-dependent fashion.

The pharmacological treatment of sleepiness in Parkinsonism has so far no apparent recommendation. Modafinil is well tolerated in PD and is frequently used despite the fact that two accomplished studies failed to provide conclusive evidence of the effects of modafinil treatment for EDS in PD [Hogl et al. 2002; Ondo et al. 2005]. Nocturnally administered sodium oxybate improved EDS and fatigue in PD in one open-label study [Ondo et al. 2008]. Anti-H3 antihistamines are still under investigation for this diagnostic group [Schwartz, 2011]. A high level of caution is needed in treating sleepiness in patients with PD and Parkinsonism who are experiencing cognitive decline or psychotic episodes.

\section{Multiple sclerosis}

Sleep disturbances are frequent in MS and their etiology is multifactorial. EDS can be confounded in MS with fatigue. Recent results show that the hypocretin system is intact and sleepiness is not typical in MS without hypothalamic lesions on MRI [Knudsen et al. 2008]. In contrast, patients with MS and neuromyelitis optica (Devic's disease) having hypothalamic lesions suffer from EDS. In the case of neuromyelitis optica this can be explained by an immune attack on membrane protein aquaporin 4 expressed in hypothalamic periventricular regions [Kanbayashi et al. 2009]. From one level-two study and one level-four study, modafinil, usually at $200 \mathrm{mg} /$ day, can be effective in keeping EDS under control in MS [Zifko et al. 2002]. EDS and fatigue in MS may be secondary to sleep fragmentation which is likely to be amenable to proper management. Restless legs syndrome occurs in MS patients more frequently, affecting more patients with longer disease duration and higher Expanded Disability Status Scale (EDSS) score [Vavrova et al. 2012].

\section{Traumatic brain injury}

Sleepiness belongs to common consequences of TBI. Even though sleepiness improves 'spontaneously' in many patients with TBI, about one quarter of TBI subjects remain sleepy for 6 months to 1 year after injury. EDS is associated with a low hypocretin level in cerebrospinal fluid which tends to reach normal values after 6 months [Baumann et al. 2007]. Short-term posttraumatic hypersomnia is frequent and no specific therapy is indicated. Persisting posttraumatic hypersomnia may develop after major as well as minor brain injury. Earlier case studies reported symptomatic treatment with amphetamines and methylphenidate, the more recent ones focus on modafinil. The level-two pharmacological trial did not confirm any favorable effect of modafinil in TBI [Jha et al. 2008] but the level-one trial provides evidence that modafinil (100-200 mg daily) improves posttraumatic EDS but does not improve posttraumatic fatigue [Kaiser et al. 2010].

\section{Stroke}

Poststroke EDS is a known condition depending, to a degree, on the lesion localization and its size. It may also be due to a sleep-related breathing disorder as a fairly frequent poststroke complication. Treatment of poststroke EDS which is not caused by the night sleep impairment is often doomed to failure. In individual patients, some improvement has been reported in thalamic and mesencephalic strokes treated with amphetamines, modafinil, methylphenidate, and dopaminergic agents [Bassetti, 2011].

\section{Brain tumors}

No systematic studies of sleep associated with tumors have been performed. Brain tumors can disrupt sleep-wake cycles when localized in sleepcontrol areas or by causing intracranial hypertension or both. Symptomatic narcolepsy is extremely rare. A somnolence after cerebral radiation has been described [Culebras, 2011].

\section{Inflammatory processes}

Inflammatory processes in general reduce wakefulness. This phenomenon is caused by higher inflammatory mediators such as tumor necrosis factor alpha and interleukin 1 that have some hypnotic activity [Shoham et al. 1987]. Encephalitis produces sleepiness or drowsiness in most cases according to clinical experience; studies on this 
topic are missing. There are some specific types of encephalitis affecting sleep and wake. Sleeping sickness, a meningoencephalitis caused by Trypanosoma brucei or Trypanosoma brucei gambiensae manifested by insomnia or hypersomnia, followed by psychomotor retardation, extrapyramidal symptoms, ataxia, seizures, coma and death. Surprisingly REM sleep parameters are preserved and SOREM periods were recorded [Culebras, 2011]. von Economo described encephalitis lethargica with epidemic peak around 1920. The main symptoms were insomnia or hypersomnia together with other namely oculomotor and extrapyramidal symptoms. Insomnia occurred in patients having lesions in anterior hypothalamus where hypersomnia in those with lesions in posterolateral hypothalamic region [von Economo, 1930]. This study became the basis for further neurophysiologic research of sleep and wake. Limbic encephalitis frequently induces hypersomnia and could be in some patients combined with REM sleep behavior disorder. The association with anti-Ma2 antibodies and low hypocretin 1 in cerebrospinal fluid was found [Compta et al. 2007].

\section{Hypersomnia due to drug or substance}

ICSD2 classification reserves this category for patients with EDS that is believed to be subsequent to substance use. This category covers hypersomnias associated with tolerance to or withdrawal from various prescription or street drugs and alcohol [American Academy of Sleep Medicine, 2005a]. The description of this category goes far beyond the scope of this article and deserves separate review.

\section{Conclusion}

Primary hypersomnias are neurological diseases reducing quality of life not only by EDS but also by other symptoms. The symptoms of primary hypersomnias are treatable and should be actively searched for and examined in appropriate ways, despite the fact that some of the diagnostic entities' causes and mechanisms remain unclear and some described treatment options could require caution. Research in all of these areas goes on, but our attention must also be focused on the daily problems of our current patients.

\section{Funding}

This work was supported by the Czech Ministry of Health (grant number IGA NT13238) and
Charles University in Prague (grant number PRVOUK P26/LF1/4).

\section{References}

Ali, M., Auger, R., Slocumb, N. and Morgenthaler, T. (2009) Idiopathic hypersomnia: clinical features and response to treatment. F Clin Sleep Med 5: 562-568.

American Academy of Sleep Medicine (2005a) The International Classification of Sleep Disorders: Diagnostic and Coding Manual, 2nd edn. Westchester, Ill.: American Academy of Sleep Medicine.

American Academy of Sleep Medicine (2005b) Practice parameters for clinical use of the Multiple Sleep Latency Test and the Maintenance of Wakefulness Test. Sleep 28: 113-121.

Arnulf, I., Zeitzer, J., File, J., Farber, N. and Mignot, E. (2005) Kleine-Levin syndrome: a systematic review of 186 cases in the literature. Brain 128: 2763-2776.

Baier, P., Hallschmid, M., Seeck-Hirschner, M., Weinhold, S., Burkert, S., Diessner, N. et al. (2011) Effects of intranasal hypocretin-1 (orexin A) on sleep in narcolepsy with cataplexy. Sleep Med 12: 941-946.

Bassetti, C. (2011) Sleep and stroke. In: Kryger, M., Roth T. and Dement, W. (eds), Principles and Practice of Sleep Medicine, 5th edn. St.Louis: Elsevier Saunders, pp. 830-993.

Baumann, C., Werth, E., Stocker, R., Ludwig, S. and Bassetti, C. (2007) Sleep-wake disturbances 6 months after traumatic brain injury: a prospective study. Brain 130: $1873-1883$.

Billiard, M., Bassetti, C., Dauvilliers, Y., DolencGroselj, L., Lammers, G., Mayer, G. et al. (2006) EFNS guidelines on management of narcolepsy. Eur $\mathcal{F}$ Neurol 13: 1035-1048.

Billiard, M., Besset, A., Montplaisir, J., Laffont, F., Goldenberg, F., Weill, J. et al. (1994) Modafinil: a double-blind multicentric study. Sleep 17(8 Suppl.): S107-S112.

Billiard, M., Jaussent, I., Dauvilliers, Y. and Besset, A. (2011) Recurrent hypersomnia: a review of 339 cases. Sleep Med Rev 15: 247-257.

Black, J. and Houghton, W. (2006) Sodium oxybate improves excessive daytime sleepiness in narcolepsy. Sleep 29: 939-946.

Black, J., Pardi, D., Hornfeldt, C. and Inhaber, N. (2010) The nightly use of sodium oxybate is associated with a reduction in nocturnal sleep disruption: a double-blind, placebo-controlled study in patients with narcolepsy. $\mathcal{F}$ Clin Sleep Med 6: 596-602. 
Boscolo-Berto, R., Viel, G., Montagnese, S., Raduazzo, D., Ferrara, S. and Dauvilliers, Y. (2011) Narcolepsy and effectiveness of gammahydroxybutyrate (GHB): A systematic review and meta-analysis of randomized controlled trials. Sleep Med Rev, in press.

Broughton, R., Fleming, J., George, C., Hill, J., Kryger, M., Moldofsky, H. et al. (1997) Randomized, double-blind, placebo-controlled crossover trial of modafinil in the treatment of excessive daytime sleepiness in narcolepsy. Neurology 49: 444-451.

Carskadon, M., Dement, W., Mitler, M., Roth, T., Westbrook, P. and Keenan, S. (1986) Guidelines for the Multiple Sleep Latency Test (MSLT): a standard measure of sleepiness. Sleep 9: 519-524.

Coelho, F., Georgsson, H. and Murray, B. (2011) Benefit of repeat Multiple Sleep Latency Testing in confirming a possible narcolepsy diagnosis. $\mathcal{F}$ Clin Neurophysiol 28: 412-414.

Compta, Y., Iranzo, A., Santamaria, J., Casamitjana, R. and Graus, F. (2007) REM sleep behavior disorder and narcoleptic features in anti-Ma2-associated encephalitis. Sleep 30: 767-769.

Culebras, A. (2011) Other neurologic disorders. In: Kryger, M., Roth, T. and Dement W. (eds), Principles and Practice of Sleep Medicine. St.Louis: Elsevier Saunders, pp. 1064-1074.

Dauvilliers, Y., Abril, B., Mas, E., Michel, F. and Tafti, M. (2009) Normalization of hypocretin-1 in narcolepsy after intravenous immunoglobulin treatment. Neurology 73: 1333-1334.

Dauvilliers, Y., Arnulf, I. and Mignot, E. (2007) Narcolepsy with cataplexy. Lancet 369: 499-511.

European Medicines Agency. (2005) Sodium Oxybate. Available at: http://www.ema.europa. eu/ema/index.jsp?curl=pages/medicines/human/ medicines/000593/human_med_001163. jsp\&mid=WC0b01ac058001d124.

Food and Drug Administration (2012) Drug Information: Modafinil. Available at: http://www. accessdata.fda.gov/drugsatfda_docs/label/2007/02071 7s020s013s018lbl.pdf?utm_campaign $\% 20 /$.

Frucht, S., Rogers, J., Greene, P., Gordon, M. and Fahn, S. (1999) Falling asleep at the wheel: motor vehicle mishaps in persons taking pramipexole and ropinirole. Neurology 52: 1908-1910.

Harsh, J., Hayduk, R., Rosenberg, R., Wesnes, K., Walsh, J., Arora, S. et al. (2006) The efficacy and safety of armodafinil as treatment for adults with excessive sleepiness associated with narcolepsy. Curr Med Res Opin 22: 761-774.

Hogl, B., Saletu, M., Brandauer, E., Glatzl, S., Frauscher, B., Seppi, K. et al. (2002) Modafinil for the treatment of daytime sleepiness in Parkinson's disease: a double-blind, randomized, crossover, placebo-controlled polygraphic trial. Sleep 25: 905-909.

Ivanenko, A. (2008) Sleep and Psychiatric Disorders in Children and Adolescents. London: Informa Healthcare. http://www.loc.gov/catdir/enhancements/ fy0806/2007049955-d.html.

Jha, A., Weintraub, A., Allshouse, A., Morey, C., Cusick, C., Kittelson, J. et al. (2008) A randomized trial of modafinil for the treatment of fatigue and excessive daytime sleepiness in individuals with chronic traumatic brain injury. $\mathcal{F}$ Head Trauma Rehabil 23: 52-63.

Johns, M. (1991) A new method for measuring daytime sleepiness: the Epworth sleepiness scale. Sleep 14: 540-545.

Kaiser, P., Valko, P., Werth, E., Thomann, J., Meier, J., Stocker, R. et al. (2010) Modafinil ameliorates excessive daytime sleepiness after traumatic brain injury. Neurology 75: 1780-1785.

Kanbayashi, T., Shimohata, T., Nakashima, I., Yaguchi, H., Yabe, I., Nishizawa, M. et al. (2009) Symptomatic narcolepsy in patients with neuromyelitis optica and multiple sclerosis: new neurochemical and immunological implications. Arch Neurol 66: 1563-1566.

Knudsen, S., Jennum, P., Korsholm, K., Sheikh, S., Gammeltoft, S. and Frederiksen, J. (2008) Normal levels of cerebrospinal fluid hypocretin-1 and daytime sleepiness during attacks of relapsing-remitting multiple sclerosis and monosymptomatic optic neuritis. Mult Scler 14: 734-738.

Kornum, B., Faraco, J. and Mignot, E. (2011) Narcolepsy with hypocretin/orexin deficiency, infections and autoimmunity of the brain. Curr Opin Neurobiol 21: 897-903.

Lankford, D. (2008) Armodafinil: a new treatment for excessive sleepiness. Expert Opin Investig Drugs 17: 565-573.

Lavault, S., Dauvilliers, Y., Drouot, X., LeuSemenescu, S., Golmard, J., Lecendreux, M. et al. (2011) Benefit and risk of modafinil in idiopathic hypersomnia vs. narcolepsy with cataplexy. Sleep Med 12: 550-556.

Lecendreux, M., Bruni, O., Franco, P., Gringras, P., Konofal, E., Nevsimalova, S. et al. (2012) Clinical experience suggests that modafinil is an effective and safe treatment for paediatric narcolepsy. 7 Sleep Res, in press.

Littner, M., Johnson, S., McCall, W., Anderson, W., Davila, D., Hartse, S. et al. (2001) Practice parameters for the treatment of narcolepsy: an update for 2000. Sleep 24: 451-466. 
Mignot, E., Hayduk, R., Black, J., Grumet, F. and Guilleminault, C. (1997) HLA DQB1*0602 is associated with cataplexy in 509 narcoleptic patients. Sleep 20: 1012-1020.

Mignot, E., Lammers, G., Ripley, B., Okun, M., Nevsimalova, S., Overeem, S. et al. (2002) The role of cerebrospinal fluid hypocretin measurement in the diagnosis of narcolepsy and other hypersomnias. Arch Neurol 59: 1553-1562.

Morgenthaler, T., Kapur, V., Brown, T., Swick, T., Alessi, C., Aurora, R. et al. (2007) Practice parameters for the treatment of narcolepsy and other hypersomnias of central origin. Sleep 30: 1705-1711.

Ondo, W., Fayle, R., Atassi, F. and Jankovic, J. (2005) Modafinil for daytime somnolence in Parkinson's disease: double blind, placebo controlled parallel trial. F Neurol Neurosurg Psychiatry 76: 1636-1639.

Ondo, W., Perkins, T., Swick, T., Hull, K., Jr, Jimenez, J., Garris, T. et al. (2008) Sodium oxybate for excessive daytime sleepiness in Parkinson disease: an open-label polysomnographic study. Arch Neurol 65: 1337-1340.

Ozaki, A., Inoue, Y., Hayashida, K., Nakajima, T., Honda, M., Usui, A. et al. (2012) Quality of life in patients with narcolepsy with cataplexy, narcolepsy without cataplexy, and idiopathic hypersomnia without long sleep time: Comparison between patients on psychostimulants, drug-naive patients and the general Japanese population. Sleep Med 13: 200-206.

Poppe, M., Friebel, D., Reuner, U., Todt, H., Koch, R. and Heubner, G. (2003) The Kleine-Levin syndrome - effects of treatment with lithium. Neuropediatrics 34: 113-119.

Roth, T., Schwartz, J., Hirshkowitz, M., Erman, M., Dayno, J. and Arora, S. (2007) Evaluation of the safety of modafinil for treatment of excessive sleepiness. F Clin Sleep Med 3: 595-602.

Schwartz, J. (2011) The histamine H3 receptor: from discovery to clinical trials with pitolisant. Br F Pharmacol 163: 713-721.

Shoham, S., Davenne, D., Cady, A., Dinarello, C. and Krueger, J. (1987) Recombinant tumor necrosis factor and interleukin 1 enhance slow-wave sleep. Am $\mathcal{F}$ Physiol 253: R142-R149.

Sonka, K., Kemlink, D., Buskova, J., Pretl, M., Srutkova, Z., Maurovich Horvat, E. et al. (2010) Obesity accompanies narcolepsy with cataplexy but not narcolepsy without cataplexy. Neuro Endocrinol Lett 31: 631-634.

US Modafinil in Narcolepsy Multicenter Study Group (1998) Randomized trial of modafinil for the treatment of pathological somnolence in narcolepsy. Ann Neurol 43: 88-97.

US Modafinil in Narcolepsy Multicenter Study Group (2000) Randomized trial of modafinil as a treatment for the excessive daytime somnolence of narcolepsy. Neurology 54: 1166-1175.

Vavrova, J., Kemlink, D., Sonka, K., Havrdova, E., Horakova, D., Pardini, B. et al. (2012) Restless legs syndrome in Czech patients with multiple sclerosis: an epidemiological and genetic study. Sleep $M e d$, in press.

Vernet, C., Leu-Semenescu, S., Buzare, M. and Arnulf, I. (2010) Subjective symptoms in idiopathic hypersomnia: beyond excessive sleepiness. F Sleep Res 19: 525-534.

von Economo, C. (1930) Sleep as a problem of localisation. F Nerv Ment Dis 71: 249-259.

Winkelman, J. and Plante, D. (2010) Foundations of Psychiatric Sleep Medicine. Cambridge: Cambridge University Press.

Wise, M., Arand, D., Auger, R., Brooks, S. and Watson, N. (2007) Treatment of narcolepsy and other hypersomnias of central origin. Sleep 30: 1712-1727.

Xyrem International Study Group (2005) Further evidence supporting the use of sodium oxybate for the treatment of cataplexy: a double-blind, placebocontrolled study in 228 patients. Sleep Med 6: 415-421.

Xyrem Multicenter Study Group (2002) A randomized, double blind, placebo-controlled multicenter trial comparing the effects of three doses of orally administered sodium oxybate with placebo for the treatment of narcolepsy. Sleep 25: 42-49.

Xyrem Multicenter Study Group (2003) A 12-month, open-label, multicenter extension trial of orally administered sodium oxybate for the treatment of narcolepsy. Sleep 26: 31-35.

Xyrem Multicenter Study Group (2004) Sodium oxybate demonstrates long-term efficacy for the treatment of cataplexy in patients with narcolepsy. Sleep Med 5: 119-123.

Zifko, U., Rupp, M., Schwarz, S., Zipko, H. and Maida, E. (2002) Modafinil in treatment of fatigue in multiple sclerosis. Results of an open-label study. f Neurol 249: 983-987.
Visit SAGE journals online http://tan.sagepub.com

@SAGE journals 